\title{
Integrações do problema de corte de estoque com aspectos operacionais: scheduling, ciclos de serra e modos alternativos de manufatura
}

\author{
Felipe Kesrouani Lemos ${ }^{1}$ \\ FEB/UNESP, Bauru, SP \\ Adriana Cristina Cherri² \\ FC/UNESP, Bauru, SP \\ Silvio Alexandre de Araujo ${ }^{3}$ \\ IBILCE/UNESP, São José do Rio Preto, SP
}

\begin{abstract}
Resumo. O problema de corte de estoque apresenta importância reconhecida tanto no meio acadêmico, como industrial. Diversas características operacionais peculiares podem ser verificadas em diferentes sistemas de produção. Este trabalho contribui no estudo de três características operacionais e suas integrações com processos de corte unidimensionais, motivada por problemas reais: (i) minimização de atraso dos itens a serem cortados; (ii) minimização de ciclos de serra; (iii) e minimização de custos com modos alternativos de produção. Para cada um dos problemas foram apresentadas a motivação real de estudo, uma revisão de literatura específica para o problema, uma proposta de formulação matemática e um método de solução utilizando geração de colunas, com seu respectivo subproblema e resultados computacionais. Os experimentos foram realizados com instâncias reais, para reforçar a relevância do problema e em um conjunto de instâncias geradas aleatoriamente para explorar o impacto dos parâmetros nos resultados obtidos.
\end{abstract}

Palavras-chave. Problema de corte de estoque, Restriçoes operacionais, Geração de colunas.

\section{Introdução}

Problemas de corte e empacotamento buscam o posicionamento sem sobreposição de itens demandados, nos limites dos objetos estocados, sendo o Problema do Corte de Estoque (PCE) relacionado à minimização da matéria-prima quando há homogeneidade dos itens cortados [6].

A imersão na indústria motiva o estudo de diferentes objetivos e restrições, com especificidades tecnológicas e empresariais [4]. Organizar o processo para bem utilizar os recursos, respeitando suas capacidades e restrições é um desafio de indústrias com operações de corte [1].

O trabalho contribuiu com a proposição de formulações e métodos de solução, além de explorar instâncias para o PCE unidimensional integrado a características operacionais particulares. Propôs-se o estudo de três problemas: (i) minimização de atrasos; (ii) minimização do custo de utilização da máquina; e (iii) minimização de custos com modos alternativos de manufatura.

\footnotetext{
1 felipeklemos@gmail.com

2 adriana.cherri@unesp.br

3 silvio.araujo@unesp.br
} 


\section{Problema de corte de estoque e minimização de atrasos}

O primeiro problema tratou da minimização do uso de matérias-primas e atraso em processos de corte unidimensional. Foi considerado o caso em que o tempo de processamento do padrão de corte depende dos itens que o compõe. Enquanto este encontra-se inexplorado, o caso de tempos de processamentos independentes já apresenta literatura com formulações propostas [1]. A motivação industrial deste estudo foi o corte de tubos em máquinas de serra para a fabricação de treliças que compõem a estrutura de aviões agrícolas. Estas abastecem uma linha de montagem, em que os custos inerentes ao atraso são muito relevantes quando comparados com o custo de desperdício de matérias-primas, dada a grande quantidade de recursos materiais, humanos e imobilizados.

\subsection{Modelagem matemática e método de solução}

Seja um conjunto de $N I$ itens unidimensionais $i(i=1, \ldots, N I)$ em tamanho $l_{i}$, demanda $b_{i}$, data de entrega $d_{i}$ e custo por unidade de tempo de atraso de $w_{i}$. Sejam $N P$ padrões de corte $p(p=1, \ldots, N P)$ em objetos de comprimento $L$ com estoque ilimitado, custo $\theta$, tempo de processamento $p t_{p}$ proporcional ao número de itens cortados, contendo $a_{i p}$ itens do tipo $i$. O tempo de processamento de um item foi considerado unitário. Seja $X_{p k}$ um conjunto de variáveis inteiras que representam a quantidade de padrões $p$ cortados na sequência $k$ da programação $(k$ $=1, \ldots, N K)$. Entende-se por sequência um conjunto de padrões de corte alocados juntos na programação de produção, sendo que entre eles não existe prioridade de sequenciamento. Desta forma, os padrões de corte na sequência $k$ só podem ser executados após o fim dos padrões na sequência $k-1$. As variáveis binárias $Z_{i k}$ assumem valor 1 se o item $i$ já tem sua demanda atendida até a sequência $k-1$ da programação e 0 , caso contrário. Denota-se por $T_{i}$ o atraso do item $i$ decorrente da alocação de seus itens na programação.

O modelo do PCE e Programação da Produção unidimensional é exposto em (1)-(7).

$$
\begin{aligned}
& \sum_{i=1}^{N I} w_{i} T_{i}+\theta \sum_{p=1}^{N P} \sum_{k=1}^{N K} X_{p k} \\
& \text { sujeito } a: \quad \sum_{p=1}^{N P} \sum_{k=1}^{N K} a_{i p} X_{p k} \geq b_{i}, \quad i=1, \ldots, N I, \\
& T_{i} \geq \sum_{p=1}^{N P} \sum_{q=1}^{k} p t_{p} X_{p q}-d_{i}-M_{i} Z_{i k}, \quad i=1, \ldots, N I, \quad k=1, \ldots, N K, \\
& Z_{i k} \leq\left(\sum_{p=1}^{N P} \sum_{q=1}^{k-1} a_{i p} X_{p q}\right) / b_{i}, \quad i=1, \ldots, N I, \quad k=1, \ldots, N K, \\
& X_{p k} \in \mathbb{Z}^{+} \text {, } \\
& p=1, \ldots, N P, \quad k=1, \ldots, N K, \\
& Z_{i k} \in\{0,1\} \text {, } \\
& T_{i}, \in \mathbb{R}^{+} \text {, } \\
& i=1, \ldots, N I, \quad k=1, \ldots, N K \text {, } \\
& i=1, \ldots, N I \text {. }
\end{aligned}
$$

A função objetivo (1) busca a minimização dos atrasos ponderados dos itens e dos custos das matérias-primas. Em (2) garante-se que a demanda total seja atendida para todos os itens $i$. Em (3) é feita a definição do atraso $T_{i}$, limitado inferiormente pela diferença entre a soma dos tempos de processamento até uma certa sequência $k$ e a data de entrega do item $d_{i}$ sempre que $i$ ainda não tenha sido terminado até a sequência $k-1$ (ou seja, $Z_{i k}=0$ ). Em (4) estabelece-se a coerência entre $Z_{i k}$ e $X_{p k}$. O domínio das variáveis de decisão é definido em (5)-(7). 
Relaxando as condições de integralidade do modelo (1)-(7), propôs-se um procedimento de geração de colunas [2]. Para obtenção de uma solução inteira, optou-se pela heurística descrita por [5], na qual o próprio modelo (1)-(7) é resolvido, porém restrito apenas às colunas geradas durante o processo de obtenção da solução ótima contínua. Além disto, foram propostas desigualdades válidas, de forma a reduzir o espaço de soluções viáveis, sem limitar a solução ótima. Por fim, propôs-se um método de obtenção de limitante inferior utilizando programação dinâmica para melhorar a qualidade do limitante obtido pela relaxação linear.

\section{$2.2 \quad$ Resultados computacionais}

A instância real apresentada foi coletada na indústria aeronáutica que motivou o problema, em um processo de corte de tubos de alumínio especial em serra fita, com o objetivo de abastecer a linha de montagem. Na Tabela 1 compara-se o resultado do modelo com o método empírico fabril.

Tabela 1: Comparativo entre solução da otimização e solução empírica da operação.

\begin{tabular}{c|cc}
\hline Indicador & Modelo proposto & Forma atual \\
\hline Custo da solução & $\mathbf{5 9 . 5 6 5}$ & 103.076 \\
Custo do atraso total & $\mathbf{3 9 . 9 9 5}$ & 83.256 \\
Quantidade de objetos (unidades) & $\mathbf{1 . 9 5 7}$ & 1.982 \\
Aproveitamento da matéria-prima & $\mathbf{9 1 , 0 1 \%}$ & $89,87 \%$ \\
Atraso médio do itens & 1.337 & $\mathbf{1 . 1 3 8}$ \\
Atraso médio de peças de reposição & $\mathbf{7 0 6}$ & 5.620 \\
Atraso médio de itens do caminho crítico & $\mathbf{1 5 4 3}$ & 5.357 \\
\hline
\end{tabular}

Uma análise exploratória da formulação foi feita através de um conjunto de 180 instâncias geradas aleatoriamente, considerando diferentes cenários de quantidade de datas de entrega, demandas, tamanhos de itens e pesos para os atrasos das ordens. O resultado desta massa de dados mostrou que o modelo matemático resulta em um gap médio de 7,8\% em um tempo médio de 316,7.

Os principais achados estatísticos, utilizando análise de variâcia, evidenciaram influência dos tamanho dos itens, prazos médios e peso dos atrasos unitários na dificuldade das instâncias, sendo o primeiro com maior impacto no tempo computacional, número de padrões de corte gerados e ocupação média dos objetos na solução final. Já a combinação de prazo médio e pesos do custo de atraso influenciaram de maneira mais pronunciada os gaps e participação percentual dos custos de material em relação ao atraso na solução.

\section{Problema de corte de estoque e minimização dos ciclos de serra com durações variadas}

O segundo problema tratou da minimização de ciclos de serra integrado ao PCE, considerando que o custo fixo unitário de utilização da máquina para um ciclo é distinto de acordo com o padrão de corte executado. Considerou-se um tempo fixo de setup do ciclo, ao qual adicionou-se um tempo unitário para cada item que consta em um dado padrão de corte. Um ciclo de serra é o período que abarca a preparação e execução de um conjunto de padrões de corte que são executados simultaneamente [7]. As abordagens encontradas para o problema de minimização do número de ciclos de serra não consideram que diferentes padrões de corte têm tempos diferentes de execução. 


\subsection{Modelagem matemática e método de solução}

Seja um conjunto de $N I$ itens unidimensionais $i(i=1, \ldots, N I)$ em tamanho $l_{i}$ e quantidade $b_{i}$. Seja um conjunto de $N P$ padrões de corte $p(p=1, \ldots, N P)$ em objetos de comprimento $L$, em quantidade ilimitada, com custo de matéria-prima $\theta$, contendo $a_{i p}$ itens do tipo $i$. Em cada padrão de corte é gasto $\gamma_{p}$ como custo de máquina para executá-lo, sendo a soma de um custo de setup st por ciclo e outro componente de custo do tempo de corte $\gamma^{\text {un }}$ para cada item no padrão de corte. Portanto, $\gamma_{p}=s t+\sum_{i=1}^{N I} a_{i p} \gamma^{u n}$. O objetivo é atender à demanda dos NI itens, com menor custo total de matéria-prima e utilização da máquina. Seja $X_{p k}$ um conjunto de variáveis inteiras que representam a quantidade de padrões $p$ cortados simultaneamente em quantidade $k$ ( $k$ $=1, \ldots, N K)$ em cada ciclo. $N K$ é, portanto, a quantidade máxima de padrões unidimensionais cortados simultaneamente na máquina, delimitada pela capacidade física da máquina.

Propõe-se em (8)-(10) o modelo do PCE associado à minimização de ciclos de serra.

$$
\begin{array}{rlrl}
\min & \sum_{k=1}^{N K} k\left(\sum_{p=1}^{N P} \theta X_{p k}\right)+\sum_{k=1}^{N K} \sum_{p=1}^{N P} \gamma_{p} X_{p k} & \\
\text { sujeito } a: & \sum_{k=1}^{N K} k\left(\sum_{p=1}^{N P} a_{i p} X_{p k}\right) \geq b_{i}, & & \\
& X_{p k} \in \mathbb{Z}^{+}, & & p=1, \ldots, N I,
\end{array}
$$

No modelo (8)-(10), a função objetivo (8) busca a minimização da quantidade de objetos utilizados e do custo fixo de utilização da máquina. Em (9) garante-se o atendimento à demanda nas diversas configurações de ciclos possíveis pela máquina. Em (10) a variável de decisão $X_{p k}$ tem seu domínio definido. Os parâmetros $a_{i p}$ e $\gamma_{p}$ foram obtidos na geração de colunas [2].

Novamente, propôs-se a utilização de um método de solução através de geração de colunas. Para este problema, foi demonstrado que não é necessário gerar padrões de corte para cada número de objetos no padrão $k(k=1, \ldots, N K)$. A solução do subproblema para $k=N K$ garante o menor custo reduzido de uma coluna a ser adicionada. Para obtenção de uma solução inteira após o processo de geração de colunas, optou-se pela mesma heurística [5].

\subsection{Resultados computacionais}

A instância real foi coletada na indústria metal-mecânica do ramo de auto-peças, que produz peças usinadas. Estudou-se um processo de corte de tubos de aço de diâmetro de 5/4", que seguem para um processo de usinagem. Os objetos podem ser cortados em até 7 unidades simultâneas por ciclo. Na Tabela 2 compara-se o resultado do modelo com o método fabril.

Tabela 2: Comparativo entre solução otimizada e solução da lógica atual de operação.

\begin{tabular}{c|ccc}
\hline Resultado & Proposto & Empírico & PCE com ciclos completos \\
\hline Ciclos de serra (unidades) & 49 & 54 & $\mathbf{4 5}$ \\
Objetos (unidades) & $\mathbf{2 8 7}$ & 378 & 315 \\
Ocupação dos objetos (\%) & $\mathbf{9 8 , 9 9 \%}$ & $75,2 \%$ & $90,2 \%$ \\
Tempo de execução (horas) & 14,2 & 15,4 & $\mathbf{1 3 , 0}$ \\
Utilização do tempo de máquina (\%) & $83,7 \%$ & $\mathbf{1 0 0 \%}$ & $\mathbf{1 0 0 \%}$ \\
Custo da solução (R\$) & $\mathbf{3 6 . 3 3 0 , 0 0}$ & $47.596,40$ & $39.678,29$ \\
\hline
\end{tabular}


Uma análise exploratória foi feita em um conjunto de 1.710 instâncias aleatórias, explorando cenários de tamanhos de itens $\left(l_{i}\right)$, demandas $\left(b_{i}\right)$, objetos simultâneos na máquina $(N K)$ e custos $\left(\theta\right.$, st e $\left.\gamma^{u n}\right)$. O gap médio obtido foi de $6,75 \%$, e tempo computacional de 41,5 segundos.

A análise estatística mostrou (i) a influência do número de objetos a serem cortados simultaneamente na média e variabilidade dos gaps; (ii) a presença de itens pequenos impactando na quantidade de padrões de corte gerados e melhor ocupação dos objetos; e (iii) a razão entre demanda total $\left(\sum_{i} b_{i}\right)$ e o número máximo de objetos cortados simultaneamente $(N K)$ como determinante para a boa ocupação dos ciclos de serra e melhoria dos gaps. A combinação em que a abordagem proposta mais se sobressaiu a uma abordagem empírica de arredondamento de ciclos completos ocorreu com maiores custos de utilização de máquina e maior presença de itens pequenos na instância.

\section{Problema de corte de estoque com modos alternativos}

O terceiro problema tratou da minimização de uso de matérias-primas em um conjunto de produtos, considerando que cada um deles pode ser manufaturado de múltiplas maneiras distintas. Analisou-se uma fábrica de postes de concreto armado, em que diferentes armaduras longitudinais de aço levam aos mesmos requisitos de resistência, por combinações de comprimentos e espessuras de seus componentes.

Modos alternativos ocorrem quando mais de um conjunto de recursos podem ser escolhidos para executar uma mesma tarefa [3]. É comum na área de projetos, já que atividades podem ser aceleradas com a alocação de recursos para atender a um prazo. Esse tipo situação pode ocorrer em sistemas fabris, seja pela possibilidade de múltiplas combinações de classes de máquinas para executar uma ordem de produção, ou pela possibilidade de múltiplas combinações de matériasprimas, como é o caso da fabricação de postes de concreto armado.

\subsection{Modelagem matemática}

Seja $J(j \in J, j=1, \ldots, N J)$ um conjunto de ordens de produção de produtos com demanda $d_{j}$. Seja $K(k \in K, k=1, \ldots, N K)$ um conjunto de espessuras de matérias-primas que podem ser utilizadas para a fabricação das ordens $J$, com tamanhos dos objetos $L$ e custo de $\theta_{k}$. O conjunto $I(i \in I, i=1, \ldots, N I)$ representa os tamanhos de itens cortados. Seja $M_{j}\left(m \in M_{j}\right)$ conjuntos de modos alternativos para o atendimento da ordem $j$, sendo que cada modos $m$ é caracterizado pela combinação de itens unidimensionais da matéria-prima de espessura $k$ e de tamanho $l_{i}$ em quantidade $b_{i k m}$. Seja $P(p \in P, p=1, \ldots, N P)$ um conjunto de padrões de corte a serem cortados da matéria-prima $k$, caracterizados por uma quantidade $a_{i p}$ de itens do tamanho $l_{i}$.

O objetivo é atender à demanda das ordens de produção $J$, escolhendo os modos de produção mais convenientes para minimizar o custo total de matéria-prima. Para isso, deve-se determinar a quantidade de padrões de corte $p$ a serem cortados de cada matéria-prima com espessura $k$, denotada pela variável de decisão inteira $X_{p k}$. Como auxiliar, a variável de decisão inteira $Z_{j m}$ indica a quantidade de produtos do tipo $j$ que serão produzidas no modo $m\left(m \in M_{j}\right)$.

O modelo do PCE Unidimensional com Modos Alternativos está retratado em (11)-(15).

No modelo (11)-(15), a função objetivo (11) minimiza o custo total com matérias-primas. Em (12) garante-se o atendimento à demanda de produtos finais. Em (13) a demanda por produtos finais é desdobrada em demanda de itens a serem cortados, que devem ser atendidos pelos padrões de corte e espessuras escolhidas. Em (14) e (15) define-se o domínio das variáveis de decisão.

Assim como nos problemas anteriores, o metodo de geração de colunas ( [2]) foi utilizado para resolver a relaxação linear do problema (14)-(15) através de geração de colunas. Para obtenção da solução inteira após o processo de geração de colunas, optou-se novamente pela mesma heurística descrita por [5]. 


$$
\begin{aligned}
& \min \quad \sum_{k \in K} \sum_{p \in P_{k}} \theta_{k} X_{p k} \\
& \text { sujeito a: } \quad \sum_{m \in M_{j}} Z_{j m} \geq d_{j}, \quad j \in J \text {, } \\
& \sum_{p \in P_{k}} a_{i p} X_{p k} \geq \sum_{j \in J} \sum_{m \in M_{j}} b_{i k m} Z_{j m}, \quad k \in K, \quad i \in I, \\
& Z_{j m} \in \mathbb{Z}^{+}, \quad \quad j \in J, \quad m \in M_{j}, \\
& X_{p k}, \in \mathbb{Z}^{+}, \quad p \in P, \quad k \in K \text {. }
\end{aligned}
$$

\subsection{Resultados computacionais}

Uma instância real foi coletada de uma indústria de postes de concreto, baseada em um orçamento que totalizou 570 postes de 5 diferentes modelos, além de 285 dormentes e 28 cruzetas. Comparou-se o resultado obtido pelo modelo com o método empírico da fábrica, em que escolheu-se inicialmente o melhor modelo e posteriormente otimizou-se a demanda gerada pelo PCE. O resultado apresentou economia de 7,5\% em relação ao custo total de aço, diminuindo de $\mathrm{R} \$ 64.232,01$ para $\mathrm{R} \$ 59.416,65$.

Uma análise exploratória da formulação foi feita através de um conjunto de 1.215 instâncias geradas aleatoriamente, considerando diferentes cenários de quantidade de alternativas e proximidade dos custos entre as diferentes opções de matérias-primas. Todas as instâncias foram geradas com 30 produtos $(N J=30)$. O resultado desta massa de dados mostrou que o modelo matemático resultou em um gap médio de 0,61\% em um tempo médio de 114,0 segundos, incluindo a geração de colunas. A solução ótima foi encontrada em 31 instâncias, enquanto o maior gap foi de $5,61 \%$.

A análise estatística dos resultados mostrou que a presença de itens de tamanhos pequenos $\left(l_{i}\right)$ e existência de poucas opções de materiais $(N K)$ são os maiores determinantes de gaps aumentados. A comparação do modelo proposto com a abordagem empírica descrita nas instâncias geradas mostrou melhoria de custos, sobretudo com a maior presença de itens grandes.

\section{Concluões e perspectivas}

O objetivo do trabalho foi explorar a integração do problema de corte de estoque unidimensional com características operacionais particulares em processos de produção. Foram estudadas as integrações com (i) o problema de minimização de atrasos, (ii) o problema de minimização de ciclos de serra e (iii) o problema de modos alternativos de manufatura. Tais aspectos desafiam a implementação de soluções otimizadas para operações de corte, e fazem com que o melhor aproveitamento de matéria-prima não seja, necessariamente, a melhor solução para o problema.

Para cada um dos três problemas foi apresentada uma motivação real, uma revisão de abordagens encontradas na literatura para o problema particular e um modelo matemática. Como estratégia de solução, o método de geração de colunas foi utilizado juntamente com um procedimento heurístico. O método foi submetido a (i) instâncias ilustrativas, para mostrar a relevância da abordagem; (ii) instâncias reais, para comprovar os ganhos potenciais de sua utilização nas fábricas que motivaram os estudos; e (iii) a um conjunto de instâncias geradas aleatoriamente, para explorar a influência dos parâmetros de entrada nos resultados obtidos.

Do ponto de vista industrial, o trabalho contribuiu através de métodos para gerar boas soluções para os desafios apresentados, em tempo computacional razoável. As soluções mostraram melhorias significativas para as fábricas que motivaram os problemas. Além dos ganhos objetivos, é 
importante ressaltar que a rápida geração de boas soluções tem impacto organizacional positivo, pois promove maior dinamismo frente às constantes mudanças de cenário enfrentadas.

Para a literatura, as principais contribuições são: (i) modelagem dos PCE integrados a datas de entrega e ciclos de serra, considerando tempos de processamentos dos padrões de corte dependentes dos itens ${ }^{4}$, (ii) modelagem do problema de minimização de custos de corte havendo múltiplos modos alternativos de manufatura ${ }^{5}$; (iii) extensa análise exploratória do impacto dos parâmetros do problema nas soluções das instâncias.

Embora as aplicações mostradas tenham motivação em setores específicos, a utilidade dos modelos e métodos não está restrita a eles. Custos com atrasos relevantes também são encontrados em ambientes com multas elevadas, como gestão de projetos e empresas logísticas, ou mesmo empresas com forte necessidade de manutenção da imagem perante o cliente, como varejo via e-commerce. Problemas de corte considerando custos de utilização de máquina devido a ciclos de serra podem ser aplicados à conformação de plástica e de vidro. Modos múltiplos de manufatura também devem encontrar aplicação na indústria moveleria ou de embalagens.

É possível explorar a integração entre os problemas apresentados, como a consideração de datas de entregas associada a ciclos de serra ou modos múltiplos de manufatura. Outras características operacionais podem ser adicionadas, como setups dependentes de sequência, custos associados à formação de lotes, cortes bidimensionais ou irregulares. Outras extensões podem explorar melhorias nos métodos propostos, seja por reformulação, soluções heurísticas para instâncias de maior porte ou melhorias nos métodos de cálculo do limitante inferior.

\section{Referências}

[1] Arbib, C., Marinelli, F., Ventura, P. One-dimensional cutting stock with a limited number of open stacks: bounds and solutions from a new integer linear programming model, International Transactions in Operational Research, 2016. DOI: 10.1111/itor.12134.

[2] Gilmore, P.C., Gomory, R.E. A linear programming approach to the cutting stock problem - Part II, Operations research, 1963. DOI: 10.1287/opre.11.6.863.

[3] Kyriakidis, T.S., Kopanos, G.M., Georgiadis, M.C. MILP formulations for single-and multimode resource-constrained project scheduling problems, Computers 8 chemical engineering, 2016. DOI: 10.1016/j.compchemeng.2011.06.007.

[4] Tomat, L., Gradišar, M. One-dimensional stock cutting: optimization of usable leftovers in consecutive orders, Central European Journal of Operations Research, 2016. DOI: 10.1007/s10100-017-0466-y.

[5] Wäscher, G., Gau, T. Heuristics for the integer one-dimensional cutting stock problem: A computational study, Operations-Research-Spektrum, 1996. DOI: 10.1007/BF01539705.

[6] Wäscher, G. Haußner, H., Schumann, H. An improved typology of cutting and packing problems, European Journal of Operational Research, 2007. DOI: 10.1016/j.ejor.2005.12.047.

[7] Yanasse, H.H. A note on the minimization of the number of cutting cycles problem, Livro de resumos do XI Simpósio de Pesquisa Operacional e Logística da Marinha - SPOLM, 2008.

\footnotetext{
${ }^{4}$ submetida e em revisão no European Journal of Operational Research (EJOR)

5 aprovada para publicação no International Journal of Production Research (IJPR), DOI $10.1080 / 00207543.2020 .1720923$
} 